Original Research

\title{
Quadriceps Strength Influences Patient Function More Than Single Leg Forward Hop During Late-Stage ACL Rehabilitation
}

Meredith Chaput, PT ${ }^{1}$, Marcus Palimenio, PT, ATC², Brooke Farmer, MS, ATC 3 , Dimitrios Katsavelis, PhD 4 , Jennifer J. Bagwell, PT, PhD ${ }^{5}$, Kimberly A. Turman, MD ${ }^{6}$, Chris Wichman, PhD 7 , Terry L. Grindstaff, PhD, PT, ATC ${ }^{3}$ a

1 Department of Physical Therapy, Ohio University, ${ }^{2}$ Makovicka Physical Therapy, ${ }^{3}$ Department of Physical Therapy, Creighton University, 4 Department of Exercise Science and Pre-Health Professions, Creighton University, ${ }^{5}$ Department of Physical Therapy, California State University Long Beach, ${ }^{6}$ MD West ONE, 7 Department of Biostatistics, University of Nebraska Medical Center

Keywords: quadriceps strength, movement system, hop test, anterior cruciate ligament reconstruction

https://doi.org/10.26603/001c.18709

International Journal of Sports Physical Therapy

Vol. 16, Issue 1, 2021

\section{Background}

A comprehensive battery of tests are used to inform return to play decisions following anterior cruciate ligament (ACL) reconstruction. Performance measures contribute to patient function, but it is not clear if achieving symmetrical performance on strength and hop tests is sufficient or if a patient also needs to meet minimum unilateral thresholds.

\section{Hypothesis/Purpose}

To determine the association of quadriceps strength and single-leg forward hop performance with patient-reported function, as measured by the IKDC Subjective Knee Form (IKDC), during late-stage ACL rehabilitation. A secondary purpose was to determine which clinical tests were the most difficult for participants to pass.

\section{Study Design}

Descriptive Laboratory Study

\section{Methods}

Forty-eight individuals with a history of ACL-R (32 female, 16 male; mean \pm SD age $=18.0 \pm 2.7 \mathrm{y}$; height $=172.4 \pm 7.6 \mathrm{~cm}$; mass $=69.6 \pm 11.4 \mathrm{~kg}$; time since surgery $=7.7 \pm 1.8$ months; IKDC=86.8 \pm 10.6 ) completed the IKDC survey, quadriceps isometric strength, and single-leg forward hop performance. The relationship between IKDC scores and performance measures (LSI and involved limb) was determined using stepwise linear regression. Frequency counts were used to determine whether participants met clinical thresholds (IKDC $\geq 90 \%$, quadriceps and single-leg forward hop LSI $\geq 90 \%$, quadriceps peak torque $\geq 3.0 \mathrm{Nm} / \mathrm{kg}$, and single-leg forward hop $\geq 80 \%$ height for females and $\geq$ $90 \%$ height for males).

\section{Results}

Quadriceps LSI and involved limb peak torque explained 39\% of the variance in IKDC scores while measures of single-leg forward hop performance did not add to the predictive model. Nearly $90 \%$ of participants could not meet established clinical thresholds on all five tests and quadriceps strength (LSI and peak torque) was the most common unmet criteria (71\% of participants).

\section{Conclusions}

During late-stage ACL rehabilitation deficits in quadriceps strength contribute more to

\footnotetext{
a Corresponding Author:

Terry L. Grindstaff

Creighton University, School of Pharmacy \& Health Professions

Physical Therapy Department

2500 California Plaza, Omaha, NE 68178.

E-mail: GrindstaffTL@gmail.com
} 
patient function and are greater in magnitude compared to hop test performance.

\section{Level of evidence}

Cross-Sectional Study, Level 3

\section{INTRODUCTION}

Approximately 250,000 anterior cruciate ligament (ACL) injuries occur each year in the United States, mostly impacting physically active individuals ages 15 to 25 years. ${ }^{1}$ Risk of reinjury is high for the first two years following ACL reconstruction (ACL-R), ${ }^{2}$ and risk is further increased for those who demonstrate impairments and disability at return to sport. 3,4 A comprehensive battery of measures, including patient reported outcome measures, strength tests, and hop tests, are used to inform return to sport decisions. ${ }^{3-5} \mathrm{~Pa}$ tient-reported outcome measures, such as the International Knee Documentation Committee Subjective Knee Form (IKDC) are frequently used after ACL-R, 6,7 and provide insights into the patient's perception of their functional abilities ranging from activities of daily living to sport activities (e.g. squat, run, jump). ${ }^{8}$

The IKDC is used in conjunction with performance-based outcome measures, such as quadriceps strength and singleleg forward hop performance, to inform rehabilitation progression and return to sport decisions. ${ }^{3-5}$ Measures from the uninvolved limb are used as a patient specific reference standard and a limb symmetry index (LSI= involved/uninvolved) is calculated to express the magnitude of differences between limbs. An LSI greater than or equal to $90 \%$ is often used as a clinical threshold indicative of recovery ${ }^{5,9}$ and meeting these thresholds is thought to decrease the risk of re-injury. ${ }^{3,4} \mathrm{~A}$ limitation of the LSI is that it may overestimate function, ${ }^{10,11}$ specifically if the uninvolved limb (i.e. comparison limb) has a history of previous injury $^{12}$ or develops weakness due to disuse. ${ }^{10,13,14}$ Diminished performance in the uninvolved limb can result in more symmetrical LSI values, but identifies symmetrically poor performance (e.g. two symmetrically flat bike tires). It is suggested that LSI values also be interpreted in context to normative performance benchmarks ${ }^{15}$ to ensure patients achieve symmetrical performance between limbs and that the overall performance meets age, sex, and/or sport performance thresholds. Clinical thresholds have been established for both isometric quadriceps strength (3.0-3.1 Nm/ $\mathrm{kg})^{16,17}$ and single leg forward hop performance (females $80 \%$ of height, males $90 \%$ of height), ${ }^{18}$ but the incorporation of unilateral thresholds to inform return to sport decisions is limited. ${ }^{5,19-21}$

Hop performance (e.g., forward hop, timed side hop, vertical jump) and patient-reported outcome measures are relatively easy to obtain in a clinical environment at little cost and with minimal equipment. Conversely, quantifiable measures of quadriceps strength require more expensive specialized equipment (e.g., electromechanical or handheld dynamometer) that may not be available in all clinical environments. These factors likely contribute to practice patterns of clinicians which indicate only about $40 \%$ of physicians and $55 \%$ of physical therapists utilize a quantifiable measure of quadriceps strength following ACL-R while $60 \%$ to $89 \%$ of providers utilize hop tests. ${ }^{22,23}$ Since there is a moderate to good positive relationship between LSI values for quadriceps strength and hop distance $\left(\mathrm{R}^{2}=0.41\right)$ in individuals with a history of ACL- $\mathrm{R},{ }^{24}$ it is often assumed that inferences regarding quadriceps strength can be made based on hop test performance. This assumption is not correct as quadriceps strength LSI deficits are of greater magnitude when compared hop tests. ${ }^{5,19,25-28}$ Using only functional performance measures and failure to obtain quantified quadriceps strength measures limits well-informed return to sport decisions and places a greater emphasis on measures that can be influenced by compensatory movement strategies yet yield similar performance (i.e., hop distance) between limbs. ${ }^{29,30}$

Deficits in both quadriceps LSI and single-leg forward hop LSI are known to negatively impact patient-oriented outcomes following ACL- $\mathrm{R}^{24,31}$ but evidence is conflicting regarding the relationship between quadriceps strength symmetry, single-leg forward hop performance symmetry, and patient-reported function. ${ }^{20,26,32}$ While previous studies have examined the individual contribution of LSI values or unilateral performance on patient-reported outcomes, ${ }^{32}$ there is limited research examining the collective contributions of both LSI values and unilateral performance. More specifically it is not known if achieving symmetrical performance is enough or if a patient also needs to meet minimum unilateral thresholds. Better understanding the relationships between performance- and patient-oriented measures can help optimize rehabilitation approaches and maximize knee function after ACL- $\mathrm{R}$ at the time return to sport decisions are being made. Therefore, the purpose of this study was to determine the association of quadriceps strength and single-leg forward hop performance with patient-reported function, as measured by the IKDC, during late-stage ACL rehabilitation. It was hypothesized that measures of quadriceps strength (peak torque and LSI) would better predict patient-reported outcome measures than single-leg forward hop (distance and LSI). In an effort to better guide rehabilitation efforts, a secondary purpose was to determine which clinical tests were the most difficult for participants to reach established thresholds. It was hypothesized that quadriceps peak torque would be the most difficult clinical test to achieve established thresholds.

\section{METHODS}

\section{PARTICIPANTS}

This was a cross-sectional study and all data collection was completed in a university research laboratory. Forty-eight individuals with a history of ACL-R volunteered for this study (Table 1). Participants were recruited or referred from the surrounding community (physical therapy clinics, athletic training rooms, orthopedic surgeon offices) and we did not control or monitor rehabilitation approaches. Measures collected during this study were used to help inform return to sport decisions during late stages of ACL rehabil- 
Table 1: Participant demographics. Values are mean \pm standard deviation or frequency counts.

\author{
Gender \\ Age \\ Height \\ Mass \\ Time Since Surgery \\ IKDC Subjective \\ Graft Type \\ Tegner Activity Scale (pre-injury) \\ Primary Sport
}

\author{
32 female; 16 male \\ $18.0 \pm 2.7$ years \\ $172.4 \pm 7.6 \mathrm{~cm}$ \\ $69.6 \pm 11.4 \mathrm{~kg}$ \\ $7.7 \pm 1.8$ months \\ $86.8 \pm 10.6 \%$ \\ 41 hamstring; 7 bone-patellar-bone \\ $8.5 \pm 1.1$
}

Soccer $n=24$; Basketball $n=9$; Football $n=4$; Softball $n=4$; Other $n=5$ itation as requested by the participant's medical care team (physician, physical therapist, athletic trainer). Actual return to sport status was not specifically monitored. Inclusion criteria included (1) unilateral ACL-R with bone-patellar tendon-bone or semitendinosus autograft, meniscus pathology permitted, (2) $\geq 5$ months following surgical reconstruction, (3) age 14-25 (4) participation in athletics at recreational level or higher (Tegner Activity Scale Score $\geq$ 6). Exclusion criteria included (1) bilateral knee injury or (2) previous knee injury requiring surgical intervention, or other ligament injury (medial collateral, posterior collateral) that required surgical repair. All ACL revision and multiple ligament repairs were also excluded. The current study was part of two larger studies used to clinically inform return to sport decisions and was approved by the Creighton University Institutional Review Board (IRB 636803 and 928791). All participants signed an approved informed consent form, compliant with the Declaration of Helsinki, and completed a standardized health history form. Participants first completed measures of height and body mass, then performed tests to determine maximum quadriceps isometric strength followed by single-leg forward hop tests, and finished with patient-reported outcome measure (IKDC).

\section{OUTCOME MEASURES}

\section{PATIENT-REPORTED OUTCOME MEASURES}

The IKDC Subjective Knee Form was used to quantify patient-reported function and includes 18 questions related to symptoms, function, and sport activity (0-100), with higher scores indicative of better status. The IKDC has good intersession reliability (ICC $=0.9595 \% \mathrm{CI}=0.91-0.98)$ and a minimal detectable change of 8.8 points. 33

\section{QUADRICEPS STRENGTH}

Isometric quadriceps strength was assessed using an electromechanical dynamometer (Biodex System 3; Computer Sports Medicine Inc., Stoughton, MA, USA) and standardized procedures with the knee at $90^{\circ}$ knee flexion. ${ }^{34-36}$ The dynamometer was interfaced with a data acquisition system (MP150; Biopac Systems, Inc., Goleta, CA, USA) and torque data were sampled at $2000 \mathrm{~Hz}$. Quadriceps strength was measured on both limbs, with the uninvolved limb measured first. Participants performed a standardized and progressive warm-up, including submaximal and maximal isometric contractions. The maximum torque was obtained during the warm-up and used to provide visual feedback (e.g., computer monitor with torque provided in real-time) during subsequent trials. Participants were provided visual feedback of torque (90\% and $100 \%$ targets) and loud verbal encouragement to ensure maximal effort during testing. ${ }^{37}$ Following the warm-up, participants performed two trials, at maximum effort, with the average peak torque $(\mathrm{Nm})$ normalized to body mass $(\mathrm{Nm} / \mathrm{kg})$ used for data analysis. An LSI was also calculated by dividing the involved limb quadriceps peak torque by the uninvolved limb quadriceps peak torque and expressed as a percentage. Measures of quadriceps strength using an electromechanical dynamometer have good to excellent intersession reliability (ICC 0.98). ${ }^{38}$

\section{SINGLE-LEG FORWARD HOP}

Participants performed the single-leg forward hop, which requires a maximum jump for distance and a controlled single-leg landing. ${ }^{5,31}$ Participants began the forward hop in a single limb stance position where they could utilize counter arm movement and were instructed to hop as far forward as possible while maintaining a controlled singleleg landing, defined as maintaining position on a single leg for at least two seconds. An unsuccessful hop was classified by loss of balance resulting in contralateral lower extremity touchdown, either upper extremity touchdown, excessive loss of balance, additional hops upon landing, or sliding of the heel. If a hop was unsuccessful, the participant was reminded of criteria for a successful hop, and they completed additional trials until a successful hop was obtained. Participants performed three successful trials. Trial one was used as a warm-up and the maximum distance $(\mathrm{cm})$ of trials two or three was used for data analysis. Hop distance normalized to height was recorded and expressed as a percentage for data analysis. Single-leg forward hop distance was also represented as an LSI by dividing the involved limb distance by the uninvolved distance and expressed as a percentage. The single-leg forward hop has excellent between session reliability (ICC $=0.92$ to 0.95 ) and a minimal detectable change of $8 \%$ for limb symmetry index ${ }^{39}$ and $13-14$ cm for absolute distance. ${ }^{40}$ 
Table 2: Quadriceps strength and single-leg forward hop. Values are mean \pm SD. Values in parentheses are normalized to body anthropometrics.

\begin{tabular}{|c|c|c|c|c|}
\hline & Involved & Uninvolved & LSI & $\begin{array}{c}\mathrm{p}- \\
\text { value }\end{array}$ \\
\hline $\begin{array}{l}\text { Quadriceps Peak } \\
\text { Torque }\end{array}$ & $\begin{array}{c}192.8 \pm 59.3 \mathrm{Nm}(2.79 \pm 0.76 \\
\mathrm{Nm} / \mathrm{kg})\end{array}$ & $\begin{array}{c}230.1 \pm 65.8 \mathrm{Nm}(3.30 \pm 0.71 \\
\mathrm{Nm} / \mathrm{kg})\end{array}$ & $\begin{array}{l}85.5 \pm \\
19.1 \%\end{array}$ & $<.001^{*}$ \\
\hline $\begin{array}{l}\text { Single-Leg Forward } \\
\text { Hop }\end{array}$ & $144.5 \pm 39.3 \mathrm{~cm}(83.7 \pm 22.0 \%)$ & $156.3 \pm 42.3 \mathrm{~cm}(90.6 \pm 23.8 \%)$ & $\begin{array}{c}92.7 \pm \\
12.5 \% \dagger\end{array}$ & $<.001^{*}$ \\
\hline
\end{tabular}

LSI= limb symmetry index

*significant difference $(\mathrm{p} \leqslant .001)$ between involved and uninvolved limbs

$\dagger$ significant difference $(\mathrm{p}=.002)$ between quadriceps peak torque and single-leg forward hop LSI values

Table 3: Correlations between patient-reported and performance outcome measures.

\begin{tabular}{|c|c|c|c|c|}
\hline & IKDC & $\begin{array}{l}\text { Quadriceps } \\
\text { LSI }\end{array}$ & $\begin{array}{l}\text { Quadriceps Peak } \\
\text { Torque }\end{array}$ & $\begin{array}{c}\text { Single Leg Forward } \\
\text { Hop LSI }\end{array}$ \\
\hline IKDC & -- & & & \\
\hline Quadriceps LSI & $.556^{*}$ & -- & & \\
\hline Quadriceps Peak Torque (Nm/kg)- involved limb & $.554^{*}$ & $.568^{*}$ & -- & \\
\hline Single Leg Forward Hop LSI & $.342^{*}$ & $.616^{*}$ & $.516^{*}$ & -- \\
\hline $\begin{array}{l}\text { Single Leg Forward Hop (normalized to height)- } \\
\text { involved limb }\end{array}$ & $.339^{*}$ & $.483^{*}$ & $.550^{*}$ & $.414^{*}$ \\
\hline
\end{tabular}

IKDC= International Knee Documentation Committee Subjective Knee Form; LSI= limb symmetry index

* indicates significant correlation $(\mathrm{p}<0.05)$

\section{STATISTICAL ANALYSIS}

Statistical analysis was conducted using SPSS software (version 26.0 IBM SPSS Statistics; Armonk, NY, USA). Mean values and standard deviations were calculated for all variables (IKDC scores, quadriceps isometric strength [Nm/ $\mathrm{kg}]$, quadriceps LSI, single-leg forward hop distance [cm], single-leg forward hop LSI). Paired t-tests were used to determine differences in quadriceps strength and single-leg forward hop distance between the involved and uninvolved limbs and LSI values. Pearson product-moment correlation coefficients were used to determine relationships between outcome measures. Next, stepwise linear regression was used to determine the ability of quadriceps strength measures (peak torque and LSI) and single-leg forward hop measures (distance and LSI) to predict IKDC scores. Predictor variables that were significantly correlated with IKDC scores were entered into the model. Significance was determined a priori as $\mathrm{p} \leq 0.05$. Correlations were qualified as good to excellent $(r \geq 0.76)$, moderate to good ( $r=0.50-0.75)$, fair $(r=0.25-0.50)$, and little to no relationship $(r \leq 0.25) .41$ Additionally, results for each performance test were dichotomized (pass/fail) based on meeting clinical thresholds used in previous studies or clinical guidelines ( $\geq 90 \%$ IKDC score $^{17,32}$ or LSI, ${ }^{5,9}$ quadriceps peak torque $\geq 3.0 \mathrm{Nm} /$ $\mathrm{kg},{ }^{16,17}$ and single-leg forward hop $\geq 80 \%$ height for females and $\geq 90 \%$ height for males). ${ }^{18}$ These results are reported as frequency counts and used to provide additional clinical insights regarding the participants. ${ }^{15}$

\section{RESULTS}

The involved limb, relative to the uninvolved limb, had significantly less quadriceps strength and decreased single-leg forward hop distance (Table 2). Participants had quadriceps peak torque LSI values (85.5\%) that were significant lower $(p=0.002)$ than single-leg forward hop LSI values $(92.7 \%)$ (Table 2). All predictor variables were significantly correlated with IKDC scores and were entered into the regression model (Table 3). Quadriceps LSI showed the strongest association with IKDC scores and explained $31 \%$ of the variance in IKDC scores (Table 4). Involved limb quadriceps peak torque normalized to body mass added to the predictive model (8\%) explaining $39 \%$ of the variance in IKDC scores (Table 4). While single-leg forward hop distance and LSI did have a fair association with IKDC scores (Table 3), these clinical tests did not add to the predictive model.

Regarding meeting clinical thresholds, only five participants $(10.4 \%)$ met or exceeded clinical thresholds on all five tests (IKDC, quadriceps LSI, quadriceps peak torque normalized to body mass, forward hop LSI, and forward hop normalized to height) while seven participants (14.6\%) failed to meet any of the clinical thresholds (Table 5). The most common unmet criteria were related to quadriceps strength where only $37.5 \%$ met peak torque thresholds for the involved limb and $41.7 \%$ met quadriceps LSI thresholds (Table 6). 
Table 4: Regression models developed to predict IKDC Scores.

\begin{tabular}{|c|c|c|c|c|c|c|c|}
\hline Model & Variable & $\begin{array}{c}\text { Standardized } \\
\beta\end{array}$ & $p$ & $\mathbf{R}$ & $\mathrm{R}^{2}$ & $\begin{array}{c}\mathrm{R}^{2} \\
\text { Change }\end{array}$ & SE \\
\hline \multirow[t]{2}{*}{1} & Constant & & $<.001$ & .556 & .309 & .309 & .089 \\
\hline & Quadriceps LSI & .556 & & & & & \\
\hline \multirow[t]{3}{*}{2} & Constant & & $<.001$ & .627 & .393 & .084 & .085 \\
\hline & Quadriceps LSI & .356 & & & & & \\
\hline & $\begin{array}{l}\text { Quadriceps peak torque (Nm/ } \\
\text { kg) }\end{array}$ & .352 & & & & & \\
\hline
\end{tabular}

IKDC= International Knee Documentation Committee Subjective Knee Form; LSI= limb symmetry index

\section{DISCUSSION}

The current study assessed quadriceps strength, single-leg forward hop performance, and a patient-reported outcome measure during late-stage ACL rehabilitation, a time when many patients are considering return to sport. Both quadriceps LSI and hop performance LSI are known to predict patient-oriented outcomes following ACL-R. ${ }^{24,31}$ As it was hypothesized, quadriceps LSI and involved limb quadriceps peak torque normalized to body mass were the strongest predictors of IKDC scores $\left(\mathrm{R}^{2}=0.39\right)$ and single-leg forward hop LSI and involved limb hop distance normalized to height did not significantly add to the predictive model. Additionally, the most common unmet test battery criteria were related to quadriceps strength (LSI and peak torque). Furthermore, $89.6 \%$ of participants did not meet suggested clinical thresholds for return to sport ${ }^{5,9,16-18,32}$ approximately eight months following ACL-R.

When considering which clinical outcomes best predicted IKDC scores, quadriceps strength symmetry and involved limb quadriceps peak torque were better predictors while single-leg forward hop performance did not add to the predictive model. Characteristics of quadriceps strength, specifically LSI and involved limb peak torque, explained $39 \%$ of the variance in IKDC scores. Symmetry accounted for $31 \%$ of the variance in IKDC scores and peak torque added a unique contribution to the predictive model (8\%). These data highlight the clinical importance of a patient achieving both symmetrical quadriceps strength and having the capacity to produce a threshold of peak torque normalized to body mass (i.e., symmetrically strong). Understanding the collective influence of variables that contribute to function allows clinicians to better weigh the information of tests and measures that inform rehabilitation progression and return to sport decisions. While previous studies have examined the individual contribution of LSI values or unilateral performance on patient-reported outcomes, ${ }^{32}$ this study demonstrates the importance of achieving both symmetry and unilateral performance thresholds. Evidence is conflicting regarding the magnitude of the relationship between quadriceps strength and patient-reported function within the first year following ACL-R. ${ }^{20,32,42}$ The current study results demonstrated a moderate to good relationship between these measures (LSI $r=0.554$, peak torque $r=0.556$ ) compared to other studies demonstrating a little to fair
Table 5: Number of clinical criteria met during late stages of ACL rehabilitation $(n=48)$.

\begin{tabular}{ccc}
\hline Number of criteria met & Frequency & Percent \\
0 & 7 & $14.6 \%$ \\
1 & 8 & $16.7 \%$ \\
2 & 12 & $25.0 \%$ \\
3 & 6 & $12.5 \%$ \\
4 & 10 & $20.8 \%$ \\
5 & 5 & $10.4 \%$ \\
\hline
\end{tabular}

relationship (LSI $r=0.357$, peak torque $r=0.282)^{32}$ or no relationship ${ }^{20}$ despite being conducted at a similar timepoint (7-8 months post-surgery) with participants around the same average age (17-19 years). It is not clear why there is such a range for correlation coefficients. Across these studies, correlation coefficients were higher when reported average quadriceps LSI and peak torque values were highest. ${ }^{32}$ No significant relationship existed between quadriceps strength and patient-reported function in a study which demonstrated the lowest LSI (68\%) and peak torque $(1.59 \mathrm{Nm} / \mathrm{kg})$ values, but specific point estimates were not provided in the manuscript. ${ }^{20}$ It is possible that a minimum level of quadriceps symmetry and strength are necessary to contribute to patient reported outcome measures. Based on the current findings, clinicians should place greater clinical value in measures of quadriceps function, specifically symmetry between limbs and involved limb peak torque, as opposed to single leg forward hop metrics.

Measures of single-leg forward hop performance (LSI and distance) each had a fair association $(r=0.34)$ with IKDC scores (Table 3 ), but did not contribute a greater amount to the predictive model than quadriceps strength, nor did measures add a unique contribution to the predictive model. The moderate association with IKDC scores in the current study are consistent with previously reported correlation coefficients $(r=0.35)^{42,43}$ but results contrasts with a previous study ${ }^{20}$ which indicated symmetrical single-leg forward hop performance and age have been shown to predict IKDC scores $\left(\mathrm{R}^{2}=0.18\right)$ and that quadriceps $\mathrm{LSI}$ values did not add to the predictive model. A key difference is that in a study by Menzer et al. ${ }^{20}$ the average LSI (68\%) and peak 
Table 6: Clinical tests and percent of participants that achieved clinical thresholds during late stages of ACL rehabilitation.

\begin{tabular}{|c|c|c|c|}
\hline Clinical Test & Pass Criteria & $\begin{array}{l}\text { Percent that } \\
\text { Met Criteria }\end{array}$ & $\begin{array}{l}\text { Percent that Did } \\
\text { Not Meet Criteria }\end{array}$ \\
\hline IKDC & $\geq 90 \%$ & $50 \%$ & $50 \%$ \\
\hline Quadriceps LSI & $\geq 90 \%$ & $41.7 \%$ & $58.3 \%$ \\
\hline $\begin{array}{l}\text { Quadriceps Peak Torque (Nm/kg)- } \\
\text { involved limb }\end{array}$ & $\geq 3.0 \mathrm{Nm} / \mathrm{kg}$ & $37.5 \%$ & $62.5 \%$ \\
\hline Single Leg Forward Hop LSI & $\geq 90 \%$ & $64.6 \%$ & $35.4 \%$ \\
\hline $\begin{array}{l}\text { Single Leg Forward Hop (normalized } \\
\text { to height)- involved limb }\end{array}$ & $\geq 80 \%$ height females, $\geq 90 \%$ height males & $45.8 \%$ & $54.2 \%$ \\
\hline Quadriceps- Symmetrical and Strong & $\mathrm{LSI} \geq 90 \%$ and involved $\operatorname{limb} \geq 3.0 \mathrm{Nm} / \mathrm{kg}$ & $29.2 \%$ & $70.8 \%$ \\
\hline $\begin{array}{l}\text { Single Leg Forward Hop- } \\
\text { Symmetrical and Good Performance }\end{array}$ & $\begin{array}{c}\mathrm{LSI} \geq 90 \% \text { and involved limb } \geq 80 \% \text { height } \\
\text { females, } 90 \% \text { height males }\end{array}$ & $39.6 \%$ & $60.4 \%$ \\
\hline
\end{tabular}

IKDC= International Knee Documentation Committee Subjective Knee Form; LSI= limb symmetry index

torque $(1.59 \mathrm{Nm} / \mathrm{kg})$ values were substantially lower than values in the current study (Table 2). While direct comparisons cannot be made between studies, Menzer et al. ${ }^{20}$ included a potential wide range of athletic ability (preinjury level of activity not provided) and did not utilize visual feedback during isometric strength measures. Participants in the current study included recreational to collegiate athletes with a Tegner score greater than or equal to six. The current study also included the use of visual feedback which has been shown to result in higher peak torque values than trials without visual biofeedback. ${ }^{37}$ These results do not negate the importance of hop testing, but indicate in this sample of participants hop testing did not provide additional insights into IKDC scores beyond information that was already provided by quantifying quadriceps strength. Hop tests are still an important component of return to sport testing and can help predict return to previous sport ${ }^{26}$ and possibly osteoarthritis development, ${ }^{44}$ although the magnitude of the association with these outcome variables may be considered low. ${ }^{43}$ Currently, there is no gold standard battery of functional assessments to determine return to sport readiness following ACL-R, but a commonality across most test batteries is inclusion of quadriceps strength and single-leg forward hop performance. ${ }^{3-5}$ In the current study, quadriceps strength explained 39\% of the variance in IKDC scores, leaving $61 \%$ of the variance unexplained. The unexplained variance may be attributed to sex, age, graft type, additional components of testing batteries, or other unknown factors. Future studies should include measures of psychological readiness, performance on additional functional tests (e.g. side hop, triple hop), and other strength measures (e.g. hamstring, hip musculature) ${ }^{45}$

In an effort to better guide rehabilitation efficiency, a secondary purpose of this study was to determine which clinical tests were the most difficult for participants to reach established thresholds ( $\underline{\text { Table 6) }}$. While most studies have incorporated LSI thresholds to inform return to sport decisions, few have incorporated both unilateral thresholds and LSI values. ${ }^{5,19-21}$ Utilizing unilateral thresholds for both strength and functional performance measures can provide valuable clinical information, especially if the unin- volved limb (i.e. comparison limb) has a history of previous injury $^{12}$ or develops weakness due to disuse. ${ }^{10,13,14}$ Criteria that focus on achieving both unilateral performance and symmetrical performance may help address limitations of LSI values that can overestimate function ${ }^{10,11}$ and help improve clinical decision making. The most common test failure (71\% of participants) was not having quadriceps function that would be considered symmetrical and strong (Table 6). Additionally, nearly $60 \%$ of participants did not meet hop test LSI and unilateral hop test performance thresholds. Measures of quadriceps function were more difficult to achieve versus the single leg forward hop test. On average, participants in the current study had quadriceps LSI values that were $7 \%$ lower than single-leg forward hop LSI (Table 2). These findings are in agreement with previous studies that have found LSI values for hop performance are greater than LSI values for quadriceps strength. $5,19,25-28$ This suggests that quadriceps strength may be more of a rate limiting factor when compared to single leg forward hop performance, but impairments in both measures exist during late stages of ACL rehabilitation and should be addressed.

Unilateral performance was consistently a more rate limiting factor for participants versus achieving LSI thresholds (Table 6). This highlights the importance of addressing unilateral performance deficits in rehabilitation and the clinical utility to incorporate normative benchmarks for performance into return to sport testing. ${ }^{15}$ On average participants met unilateral quadriceps strength ${ }^{16,17}$ and single leg forward hop performance, ${ }^{18}$ (Table 6) benchmarks for the uninvolved limb, suggesting the capacity for adequate performance in the involved limb was available, but not achieved. A limitation of previous studies ${ }^{16,17}$ that established quadriceps strength thresholds is that they do not specifically account for sex specific or age specific differences in strength ${ }^{46,47}$ which may make application of threshold metrics confusing when applying to an individual patient (e.g. female high school soccer player versus male collegiate football player). Additionally, normative hop test thresholds need further validation as these were developed based on clinical observations. ${ }^{18}$ Future studies should bet- 
ter develop age, sex, and/or sport performance thresholds to better guide clinical decisions for individual patients.

Despite the evidence supporting the results that quadriceps strength is an essential determinant of function, it is a common clinical outcome that goes unassessed. ${ }^{22,23}$ While there is a moderate to good relationship between measures of involved limb quadriceps peak torque and single leg forward hop distance $(r=0.55)$ as well as a moderate to good relationship between the associated LSI values ( $\mathrm{r}=$ 0.62 ), caution should be exercised in assuming that individuals with more symmetrical single-leg forward hop performance therefore have adequate quadriceps strength. It is not clear why LSI values for single leg forward hop performance were significantly greater than LSI values for quadriceps strength. It is possible individuals shorted hop distance on the contralateral limb or employed different hop strategies (e.g., trunk position, increased contributions from the hip and ankle) between limbs to achieve more symmetrical performance. ${ }^{29,30}$ This is a limitation of clinical hop test measures since it is difficult to determine specific hop strategies without biomechanical testing. Biomechanical testing would provide insights into joint specific contributions to hop tests performance. Since this technology is often not available in clinical settings, this further strengthens the rationale to obtain quantifiable measures of both quadriceps strength (joint specific function) and single-leg forward hop performance (lower extremity function). Obtaining lower extremity strength measures in a clinical environment can be challenging without access to an electromechanical dynamometer, which requires extensive training, practice, time, and cost. A hand-held dynamometer offers a less expensive, user-friendly, option to quantify quadriceps isometric strength in a clinical environment and is a valid measure $(r=0.89-0.93)$ when compared to a gold standard electromechanical dynamometer. ${ }^{35,36}$ Future research should examine the clinical utility of an hand-held dynamometer to assess quadriceps strength in individuals returning to activity following ACL$\mathrm{R}$, as the current results demonstrate an ongoing need for this clinical assessment feasibility.

Utilizing a battery of tests, including functional performance, strength, and patient-reported outcome measures, is the recommended standard to inform rehabilitation progression and return to sport decisions. ${ }^{3-5}$ Early identification of deficits is important since athletes not meeting return to sport criteria ( $\geq 90 \%$ LSI quadriceps strength, hop tests, patient-reported outcomes) and returning to higherlevel activities are at a significantly greater risk of re-injury (4-5x more likely). ${ }^{3,4}$ Additionally, individuals who do not pass criteria at 6 months are about twice as likely to fail return to sport criteria 12 and 24 months following ACLR. ${ }^{26}$ A substantial concern from the current study population, in late stages of ACL rehabilitation, is that only $10.4 \%$ of participants (5 of 48) met benchmarks for IKDC scores, quadriceps strength (LSI and peak torque), and single-leg forward hop (LSI and normalized hop distance) and 14.6\% (7 of 48) did not meet any of the five clinical criteria used to inform return to sport decisions (failed all 5 criteria). Rates for passing return to sport criteria within the first year following ACL-R vary from $7-58 \% .5,21,25,26,48$ The wide range of differences between studies may be due to the thresholds and outcomes used to inform return to sport decisions, ${ }^{5}$ population investigated, ${ }^{25,48}$ and control of pre and postoperative rehabilitation. ${ }^{26}$ Previous studies have used a cutoff as low as $76 \%$ for IKDC scores ${ }^{20}$ and $80-85 \%$ for quadriceps strength and single leg forward hop LSI values. ${ }^{13,49}$ While these studies have selected relatively low thresholds other studies have suggested that IKDC scores, ${ }^{32,50}$ quadriceps LSI, ${ }^{17}$ and single leg forward hop LSI $^{20}$ should be higher (e.g. $\geq 92-95 \%$ ). Higher thresholds or more stringent criteria would make overall pass rates lower, but may help ensure athletes are indeed ready to return to activity. Studies which derive participants from a health registry 48 or large metropolitan area, ${ }^{25}$ without control of post-operative rehabilitation, have relatively low rates $(14-30 \%)^{25,48}$ of individuals who pass return to sport criteria compared to higher rates (50-58\%) of individuals who pass return to sport criteria from studies with greater control of post-operative rehabilitation. ${ }^{26}$ The current study demonstrates a population of individuals recovering from ACL-R from a variety of local physical therapy clinics and surgery performed by several area orthopedic surgeons. These results likely reflect the variance in clinical practice and factors used to inform rehabilitation progression or return to sport decisions. The findings are consistent with studies of similar design which demonstrates the need for more consistent and improved rehabilitation strategies. ${ }^{25,48}$ Supervised rehabilitation, performed greater than 6 months in duration, has been shown to result higher LSI values for strength and hop test performance. ${ }^{27}$

A limitation of this study was that, although the results were obtained during late-stage ACL rehabilitation, athlete status of actual return to sport or previous level of play (e.g., starter versus secondary), contact hours per week, and type of sport were not monitored. Thus, it is unknown whether results of testing were actually used to inform return to sport decisions or if participants indeed did return to sport. Additionally, participants underwent a primary unilateral ACL-R with minimal concomitant injuries (meniscus injury permitted), thus results cannot be generalized to those with more extensive knee injuries. The current study did not control for surgical technique or graft type (bone-patellar tendon-bone or semitendinosus autograft) which is known to influence quadriceps- and hamstring strength-related outcomes differently. ${ }^{51}$ When utilizing LSI as a measure for return to sport, baseline measures prior to ACL-R can decrease overestimation of symmetry and uninvolved limb deficits that result from decreased activity; ${ }^{10}$ baseline measures (preoperative) were not available in this study. Another study limitation was the relatively limited sample size $(n=48)$ which may have decreased the statistical power and contributed to findings which are of greater magnitude than studies with larger sample sizes $(n=88-139) .{ }^{20,32}$ Despite these limitations, the current study presents real clinical applications that rehabilitation specialists face in everyday practice. Therefore, the conclusion and clinical implications are of great value for the present subject population and timeframe of return to sport indicated in the current study.

\section{CONCLUSION}

Limb-to-limb asymmetries are prominent in both quadri- 
ceps isometric strength and single-leg forward hop during late stages of ACL rehabilitation (5-12 months postsurgery). During late-stage ACL rehabilitation quadriceps peak torque symmetry (85.5\%) was significantly less than single-leg forward hop for distance symmetry (92.7\%). Quadriceps function (LSI and involved limb peak torque) showed the strongest association with IKDC scores (39\% variance) and single-leg forward hop (distance and LSI) measures did not add to the predictive model. Collectively this suggests that quadriceps strength is a more rate limiting factor that contributes to patient function during late stages of ACL rehabilitation when compared to measures of single leg forward hop performance. Additionally, nearly $90 \%$ of participants in this study had not achieved acceptable values for any of the clinical criteria used to inform return to sport decisions (IKDC, quadriceps strength, and hop performance $)^{5,9,16-18,32}$ within 5-12 months after ACL$\mathrm{R}$, suggesting they were not adequately prepared to return to activity/sport. The clinical relevance of this work is that, during late-stage ACL rehabilitation, clinicians should utilize a series of isolated strength measures, functional tests, and self-reported outcome measures to comprehensively assess the athlete function and objectively inform rehabilitation progression and return to sport decisions.

\section{FUNDING}

Portions of research reported in this publication was supported by the National Institute of General Medical Sciences of the National Institutes of Health under award number 5P20GM109090 (PI Stergiou)

\section{ETHICAL APPROVAL}

Study was granted by the Institutional Review Board at Creighton University (IRB 636803 and 928791). Written informed consent was obtained from each participant prior to participation.

\section{PUBLIC TRIALS REGISTRY}

NCT03132987

Submitted: December 02, 2019 CDT, Accepted: July 21, 2020 CDT 


\section{REFERENCES}

1. Sanders TL, Maradit Kremers H, Bryan AJ, et al. Incidence of anterior cruciate ligament tears and reconstruction: A 21-year population-based study. Am J Sports Med. 2016;44(6):1502-1507. doi:10.1177/0 $\underline{363546516629944}$

2. Paterno MV, Rauh MJ, Schmitt LC, Ford KR, Hewett TE. Incidence of second ACL injuries 2 years after primary ACL reconstruction and return to sport. Am J Sports Med. 2014;42(7):1567-1573. doi:10.1177/03635 46514530088

3. Grindem H, Snyder-Mackler L, Moksnes H, Engebretsen L, Risberg MA. Simple decision rules can reduce reinjury risk by $84 \%$ after ACL reconstruction: The Delaware-Oslo ACL cohort study. Br J Sports Med. 2016;50(13):804-808. doi:10.1136/bjsports-2016-0960 $\underline{31}$

4. Kyritsis P, Bahr R, Landreau P, Miladi R, Witvrouw E. Likelihood of ACL graft rupture: Not meeting six clinical discharge criteria before return to sport is associated with a four times greater risk of rupture. $\mathrm{Br}$ J Sports Med. 2016;50(15):946-951. doi:10.1136/bjspor ts-2015-095908

5. Gokeler A, Welling W, Zaffagnini S, Seil R, Padua D. Development of a test battery to enhance safe return to sports after anterior cruciate ligament reconstruction. Knee Surg Sports Traumatol Arthrosc. 2017;25(1):192-199. doi:10.1007/s00167-016-4246-3

6. Hambly K, Griva K. Ikdc or koos? Which measures symptoms and disabilities most important to postoperative articular cartilage repair patients? Am J Sports Med. 2008;36(9):1695-1704. doi:10.1177/03635 46508317718

7. Collins NJ, Misra D, Felson DT, Crossley KM, Roos EM. Measures of knee function. Arthritis Care Res. 2011;63(S11):S208-S228. doi:10.1002/acr.20632

8. Hambly K, Griva K. IKDC or KOOS: Which one captures symptoms and disabilities most important to patients who have undergone initial anterior cruciate ligament reconstruction? Am J Sports Med. 2010;38(7):1395-1404. doi:10.1177/036354650935967 $\underline{8}$

9. Logerstedt DS, Scalzitti D, Risberg MA, et al. Knee stability and movement coordination impairments: Knee ligament sprain revision 2017. J Orthop Sports Phys Ther. 2017;47(11):A1-A47. doi:10.2519/jospt.201 7.0303
10. Wellsandt E, Failla MJ, Snyder-Mackler L. Limb symmetry indexes can overestimate knee function after anterior cruciate ligament injury. J Orthop Sports Phys Ther. 2017;47(5):334-338. doi:10.2519/jospt.201 $\underline{7.7285}$

11. Gokeler A, Welling W, Benjaminse A, Lemmink K, Seil R, Zaffagnini S. A critical analysis of limb symmetry indices of hop tests in athletes after anterior cruciate ligament reconstruction: A case control study. Orthop Traumatol Surg Res. 2017;103(6):947-951. doi:10.1016/j.otsr.2017.02.015

12. Zwolski C, Schmitt LC, Thomas S, Hewett TE, Paterno MV. The utility of limb symmetry indices in return-to-sport assessment in patients with bilateral anterior cruciate ligament reconstruction. Am J Sports Med. 2016;44(8):230-238. doi:10.1177/036354651664 $\underline{5084}$

13. Thomeé R, Neeter C, Gustavsson A, et al. Variability in leg muscle power and hop performance after anterior cruciate ligament reconstruction. Knee Surg Sports Traumatol Arthrosc. 2012;20(6):1143-1151. doi:10.1007/s00167-012-191 2-y

14. Urbach D, Awiszus F. Impaired ability of voluntary quadriceps activation bilaterally interferes with function testing after knee injuries. A twitch interpolation study. Int J Sports Med. 2002;23(4):231-236. doi:10.1055/s-2002-29074

15. Thomeé R, Kaplan Y, Kvist J, et al. Muscle strength and hop performance criteria prior to return to sports after ACL reconstruction. Knee Surg Sports Traumatol Arthrosc. 2011;19(11):1798-1805. doi:10.1007/s0016 7-011-1669-8

16. Kuenze C, Hertel J, Saliba S, Diduch DR, Weltman A, Hart JM. Clinical thresholds for quadriceps assessment after anterior cruciate ligament reconstruction. J Sport Rehabil. 2015;24(1):36-46. do i:10.1123/jsr.2013-0110

17. Pietrosimone B, Lepley AS, Harkey MS, et al. Quadriceps strength predicts self-reported function post-ACL reconstruction. Med Sci Sports Exerc. 2016;48(9):1671-1677. doi:10.1249/mss.00000000000 $\underline{00946}$

18. Davies G, Zillmer DA. Functional progression of a patient through a rehabilitation program. 2000;9:103-118. 
19. Nagai T, Schilaty ND, Laskowski ER, Hewett TE. Hop tests can result in higher limb symmetry index values than isokinetic strength and leg press tests in patients following ACL reconstruction. Knee Surg Sports Traumatol Arthrosc. 2020;28(3):816-822. doi:1 $\underline{0.1007 / \mathrm{s} 00167-019-05513-3}$

20. Menzer H, Slater LV, Diduch D, et al. The utility of objective strength and functional performance to predict subjective outcomes after anterior cruciate ligament reconstruction. Orthop J Sports Med. 2017;5(12):232596711774475. doi:10.1177/232596711 $\underline{7744758}$

21. Welling W, Benjaminse A, Lemmink K, Gokeler A. Passing return to sports tests after ACL reconstruction is associated with greater likelihood for return to sport but fail to identify second injury risk. Knee. 2020;27(3):949-957. doi:10.1016/j.knee.202 $\underline{0.03 .007}$

22. Greenberg EM, Greenberg ET, Albaugh J, Storey E, Ganley TJ. Rehabilitation practice patterns following anterior cruciate ligament reconstruction: A survey of physical therapists. J Orthop Sports Phys Ther. 2018;48(10):801-811. doi:10.2519/jospt.2018.8264

23. Greenberg EM, Greenberg ET, Albaugh J, Storey E, Ganley TJ. Anterior cruciate ligament reconstruction rehabilitation clinical practice patterns: A survey of the PRISM society. Orthop J Sports Med. 2019;7(4). do i:10.1177/2325967119839041

24. Schmitt LC, Paterno MV, Hewett TE. The impact of quadriceps femoris strength asymmetry on functional performance at return to sport following anterior cruciate ligament reconstruction. J Orthop Sports Phys Ther. 2012;42(9):750-759. doi:10.2519/jos pt.2012.4194

25. Toole AR, Ithurburn MP, Rauh MJ, Hewett TE, Paterno MV, Schmitt LC. Young athletes cleared for sports participation after anterior cruciate ligament reconstruction: How many actually meet recommended return-to-sport criterion cutoffs? J Orthop Sports Phys Ther. 2017;47(11):825-833. doi:1 $\underline{0.2519 / j o s p t .2017 .7227}$

26. Nawasreh Z, Logerstedt D, Cummer K, Axe MJ, Risberg MA, Snyder-Mackler L. Do patients failing return-to-activity criteria at 6 months after anterior cruciate ligament reconstruction continue demonstrating deficits at 2 years? Am J Sports Med. 2017;45(5):1037-1048. doi:10.1177/036354651668061 9

27. Ebert JR, Edwards P, Yi L, et al. Strength and functional symmetry is associated with postoperative rehabilitation in patients following anterior cruciate ligament reconstruction. Knee Surg Sports Traumatol Arthrosc. 2018;26(8):2353-2361. doi:10.100 7/s00167-017-4712-6
28. Norte GE, Goetschius JW, Slater LV, Hart JM. Influence of patient demographics and surgical characteristics on pass rates of return-to-activity tests in anterior cruciate ligament-reconstructed patients before physician clearance. Clin J Sport Med. February 2020. doi:10.1097/jsm.0000000000000790

29. Ernst GP, Saliba E, Diduch DR, Hurwitz SR, Ball DW. Lower-extremity compensations following anterior cruciate ligament reconstruction. Phys Ther. 2000;80(3):251-260. doi:10.1093/ptj/80.3.251

30. Orishimo KF, Kremenic IJ, Mullaney MJ, McHugh MP, Nicholas SJ. Adaptations in single-leg hop biomechanics following anterior cruciate ligament reconstruction. Knee Surg Sports Traumatol Arthrosc. 2010;18(11):1587-1593. doi:10.1007/s00167-010-118 $\underline{5-2}$

31. Logerstedt D, Grindem H, Lynch A, et al. Singlelegged hop tests as predictors of self-reported knee function after anterior cruciate ligament reconstruction: The Delaware-Oslo ACL cohort study. Am J Sports Med. 2012;40(10):2348-2356. doi:10.1177/ 0363546512457551

32. Zwolski C, Schmitt LC, Quatman-Yates C, Thomas $S$, Hewett TE, Paterno MV. The influence of quadriceps strength asymmetry on patient-reported function at time of return to sport after anterior cruciate ligament reconstruction. Am J Sports Med. 2015;43(9):2242-2249. doi:10.1177/036354651559125 $\underline{8}$

33. Crawford K, Briggs KK, Rodkey WG, Steadman JR. Reliability, validity, and responsiveness of the IKDC score for meniscus injuries of the knee. Arthroscopy. 2007;23(8):839-844. doi:10.1016/j.arthro.2007.02.005

34. Grindstaff TL, Threlkeld AJ. Optimal stimulation parameters to detect deficits in quadriceps voluntary activation. J Strength Cond Res. 2014;28(2):381-389. ㅁ oi:10.1519/jsc.0b013e3182986d5f

35. Hansen EM, McCartney CN, Sweeney RS, Palimenio MR, Grindstaff TL. Hand-held dynamometer positioning impacts discomfort during quadriceps strength testing: A validity and reliability study. Int J Sports Phys Ther. 2015;10(1):62-68.

36. Lesnak J, Anderson D, Farmer B, Katsavelis D, Grindstaff TL. Validity of hand-held dynamometry in measuring quadriceps strength and rate of torque development. Intl J Sports Phys Ther. 2019;14(2):180-187. doi:10.26603/ijspt20190180

37. Luc BA, Harkey MH, Arguelles GD, Blackburn JT, Ryan ED, Pietrosimone B. Measuring voluntary quadriceps activation: Effect of visual feedback and stimulus delivery. J Electromyogr Kinesiol. 2016;26:73-81. doi:10.1016/i.jelekin.2015.10.006 
38. Grindstaff TL, Palimenio MR, Franco M, Anderson D, Bagwell JJ, Katsavelis D. Optimizing betweensession reliability for quadriceps peak torque and rate of torque development measures. Journal of Strength and Conditioning Research. 2019;33(7):1840-1847. do i:10.1519/jsc.0000000000002821

39. Reid A, Birmingham TB, Stratford PW, Alcock GK, Giffin JR. Hop testing provides a reliable and valid outcome measure during rehabilitation after anterior cruciate ligament reconstruction. Phys Ther. 2007;87(3):337-349. doi:10.2522/ptj.20060143

40. Thomas C, Dos'Santos T, Comfort P, Jones P. Between-session reliability of common strength- and power-related measures in adolescent athletes. Sports. 2017;5(1):15.

41. Portney LG, Watkins MP. Foundations of Clinical Research: Applications to Practice. 3rd ed. Upper Saddle River, NJ: Pearson Prentice Hall; 2009.

42. Müller U, Krüger-Franke M, Schmidt M, Rosemeyer B. Predictive parameters for return to preinjury level of sport 6 months following anterior cruciate ligament reconstruction surgery. Knee Surg Sports Traumatol Arthrosc. 2015;23(12):3623-3631. do i:10.1007/s00167-014-3261-5

43. Losciale JM, Bullock G, Cromwell C, Ledbetter L, Pietrosimone L, Sell TC. Hop testing lacks strong association with key outcome variables after primary anterior cruciate ligament reconstruction: A systematic review. Am J Sports Med. 2020;48(2):511-522. doi:10.1177/0363546519838794

44. Lui PPY, Cheng YY, Yung SH, Hung ASL, Chan KM. A randomized controlled trial comparing bone mineral density changes of three different ACL reconstruction techniques. Knee. 2012;19(6):779-785. doi:10.1016/i.knee.2012.02.005
45. Ardern CL, Taylor NF, Feller JA, Webster KE. Fiftyfive per cent return to competitive sport following anterior cruciate ligament reconstruction surgery: An updated systematic review and meta-analysis including aspects of physical functioning and contextual factors. Br J Sports Med. 2014;48(21):1543-1552. doi:10.1136/bjsports-2013-09 $\underline{3398}$

46. Kuenze C, Pietrosimone B, Lisee C, et al. Demographic and surgical factors affect quadriceps strength after ACL reconstruction. Knee Surg Sports Traumatol Arthrosc. 2019;27(3):921-930. doi:10.1007/ s00167-018-5215-9

47. Kong PW, Burns SF. Bilateral difference in hamstrings to quadriceps ratio in healthy males and females. Phys Ther Sport. 2010;11(1):12-17. doi:10.101 6/j.ptsp.2009.09.004

48. Beischer S, Senorski EH, Thomeé C, Samuelsson $\mathrm{K}$, Thomeé R. Young athletes return too early to kneestrenuous sport, without acceptable knee function after anterior cruciate ligament reconstruction. Knee Surg Sports Traumatol Arthrosc. 2018;26(7):1966-1974. doi:10.1007/s00167-017-474 $\underline{7-8}$

49. Barber-Westin SD, Noyes FR. Factors used to determine return to unrestricted sports activities after anterior cruciate ligament reconstruction. Arthroscopy. 2011;27(12):1697-1705. doi:10.1016/j.art hro.2011.09.009

50. Lentz TA, Zeppieri GJ, Tillman SM, et al. Return to preinjury sports participation following anterior cruciate ligament reconstruction: Contributions of demographic, knee impairment, and self-report measures. J Orthop Sports Phys Ther. 2012;42(11):893-901. doi:10.2519/jospt.2012.4077

51. Fischer F, Fink C, Herbst E, et al. Higher hamstring-to-quadriceps isokinetic strength ratio during the first post-operative months in patients with quadriceps tendon compared to hamstring tendon graft following ACL reconstruction. Knee Surg Sports Traumatol Arthrosc. 2018;26(2):418-425. doi:1 $\underline{0.1007 / \mathrm{s} 00167-017-4522-\mathrm{X}}$ 